\title{
An Integrated Physiological Model of the Lung Mechanics and Gas Exchange Using Electrical Impedance Tomography in the Analysis of Ventilation Strategies in ARDS Patients
}

\author{
M. Denaï, M. Mahfouf, A. Wang, D.A. Linkens \\ Dept of Automatic Control, University of Sheffield, Mappin Street, Sheffield, UK \\ m.denai@shef.ac.uk,m.mahfouf@shef.ac.uk,a.wang@shef.ac.uk,d.linkens@shef.ac.uk \\ G. H. Mills \\ Dept of Critical Care, Anaesthesia and Operating Services, Northern General Hospital, Sheffield, UK \\ Gary.mills@sth.nhs.uk
}

\begin{abstract}
Keywords: Electrical Impedance Tomography, EIT, medical imaging, lung mechanics, alveolus model, pressurevolume curve, blood gas model, mechanical ventilation, dead-space, shunt.

Abstract: Thoracic Electrical Impedance Tomography (EIT) is a non-invasive technique which attempts to reconstruct a cross-sectional image of the internal spatial distribution of conductivity from electrical measurements made by injecting small alternating currents via an electrode array placed on the surface of the thorax. Because air is highly resistive to electric currents whereas fluids and blood are good conductors, it is possible to detect changes in lungs air content with EIT enabling the assessment of ventilation distribution. This paper presents a physiological model which integrates a previously developed gas exchange model with a model of the lung mechanics. This model is combined with a two-dimensional (2D) finite element mesh of the thorax to simulate EIT image reconstruction in patients with acute Respiratory Distress Syndrome (ARDS) under mechanical ventilation. The model was able to track lung ventilation distribution under various simulated ARDS conditions and ventilator settings.
\end{abstract}

\section{INTRODUCTION}

Mechanical ventilation is an essential component in supportive therapy of patients with Acute Respiratory Distress Syndrome (ARDS): a potentially severe form of respiratory failure.

Although, mechanical ventilation can be a lifesaving intervention for many patients in the Intensive Care Unit (ICU), it has been associated with potential complications causing secondary lung damage known as Ventilator-Induced Lung Injury (VILI) (Tremblay and Slutsky, 2006). Selecting appropriate ventilator settings can reduce the risk of VILI. However, known bedside measures to guide the clinician when adjusting mechanical ventilation to provide adequate gas exchange whilst minimising any adverse effects to the patient's lungs are limited. Current methods available for assessing the lung function in mechanically ventilated patients include arterial blood gas analysis and graphic waveforms displayed on the ventilators (flow, pressure, volume over time as well as airway pressure-volume curves). However, these can give only an indication of the overall lung function and fail to provide full information on the regional lung behaviour.

Currently, chest Computed Tomography (via a CT scanner) is the most reliable technique for the clinical assessment of regional lung recruitment and ventilation distribution in patients with ARDS. By measuring lung densities, well-aerated regions can be easily differentiated from poorly and/or nonaerated lung regions. However, CT exposes the patient to radiations and is not a routine bedside technique. Ideally, mechanical ventilation should be conducted with sufficient Peak End-Expiratory Pressure (PEEP) to achieve adequate oxygenation and prevent end- expiratory collapse and with a small tidal volume to avoid end-inspiratory overdistension. However, due to the absence of bedside monitoring of regional lung ventilation, the choice of optimal ventilator titration strategy remains controversial (Levy, 2002). 
The aim of Electrical Impedance Tomography (EIT) (Brown and Barber, 1992) is to produce a cross-sectional image of the internal distribution of conductivity, or alternatively resistivity of the lungs from electrical measurements made by injecting small alternating current patterns via surface electrodes and recording the resulting boundary voltages. Because air has a high electrical resistivity compared to other lung tissues, EIT offers a very promising tool for monitoring the pulmonary function. EIT suffers from some limitations that may prevent its adoption for routine medical diagnosis. Its major limitations are low spatial resolution, susceptibility to noise and electrode errors, and in medical imaging, large variability of images between subjects. Therefore, EIT is not suitable for anatomical imaging in the way Magnetic Resonance Imaging (MRI) or CT scanners are. EIT does however, have some advantages such as being relatively inexpensive, non-cumbersome, noninvasive and radiation-free. Additionally, it has the ability to produce a high number of frames per second which makes it very useful for continuous real-time lung imaging.

The first clinical images obtained with EIT were produced by the Sheffield group (Brown et al., 1985), who developed a system that used the electrical impedance of various tissues within the human body to produce tomographic image maps of the resistivity distribution. Among the clinical applications of EIT being currently investigated by other groups one can cite the monitoring of internal bleeding, studies relating to the emptying of stomach, the monitoring of the heart function and brain activities for improving electro-cardiograms (EEG) and electro- encephalograms (EEG). However, the monitoring of the pulmonary function is considered as the most promising application of EIT which is expected to become, in the future, a valuable bedside tool in respiratory ICU. The Sheffield Research Group produced the first images of the pulmonary function using a simple backprojection algorithm to reconstruct cross-section images of the thorax (Brown et al., 1985). The functional image showed an increase in resistivity during spontaneous inspiration of the subject. A filtered-back-projection method, similar to the one used in X-ray CT imaging was later implemented by the same group to improve the spatial resolution of the reconstructed images.

Many current ongoing research studies are being directed at demonstrating the ability of EIT to image regional lung ventilation in a clinical setting (Victorino et al., 2004; Hinz et al., 2005; Putensen et al., 2007). Comprehensive literature reviews in this field can be found in (Frerichs et al., 2000) and
(Panoutsos et al., 2007). Recently a software package (EIDORS) implementing different methods for the solution of the forward and inverse problems in EIT using finite elements modelling techniques has been made available for public use (Adler and Lionheart, 2006).

The purpose of this study is to present a comprehensive physiological model of patients under mechanical ventilation. The model combines a blood gas model (SOPAVent) (Wang et al., 2006) and a model of the lung mechanics with a 2D finite element model of the thorax to simulate EIT current injection and image reconstruction. The physiological model is intended to provide the foundation for the validation of a new EIT-based clinical decision support system for optimising mechanical ventilator settings in ARDS patients.

The rest of the paper is organised as follows. Section 2 focuses on the description of the physiological model and its principal components. The approach used to combine SOPAVent model with the lung mechanics and EIT is presented. Section 3 presents a simulation study with constructed scenarios of ARDS lungs.

\section{OVERVIEW OF THE PHYSIOLOGICAL MODEL}

The structure of the simulation model is depicted in Fig. 1.

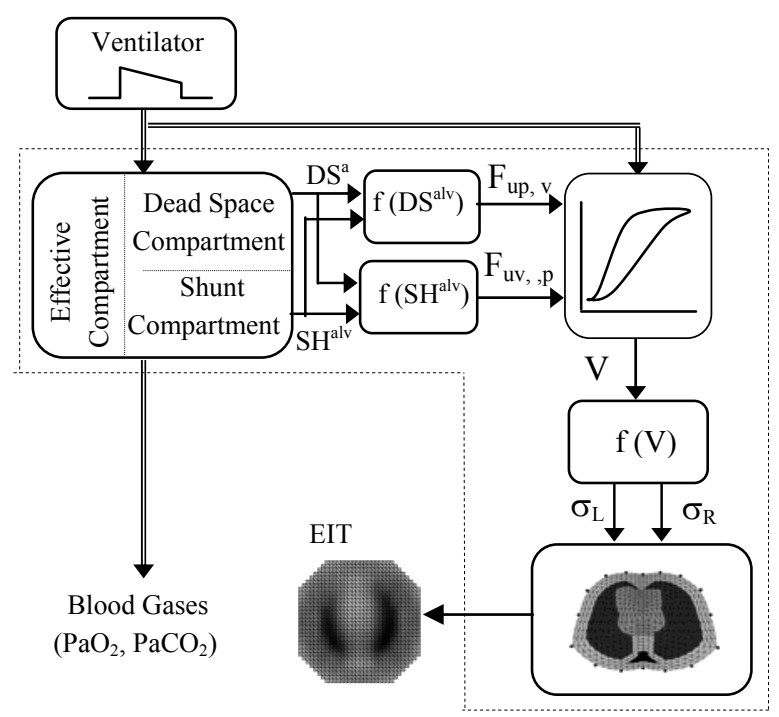

Figure 1: Schematic overview of the simulation model.

The model inputs are the ventilator parameters: the Fraction of Inspired Oxygen $\left(\mathrm{FiO}_{2}\right)$, the Tidal Volume $\left(\mathrm{V}_{\mathrm{T}}\right)$, the Peak End-Expiratory Pressure 
(PEEP), the Peak Inspiratory Pressure (PIP), the Respiratory Rate (RR), the inspiration to expiration time ratio (I:E). The outputs are the predicted blood gases: the arterial partial pressure of oxygen $\left(\mathrm{PaO}_{2}\right)$, the arterial partial pressure of carbon dioxide $\left(\mathrm{PaCO}_{2}\right)$ and EIT image of the lung resistivity distribution.

\subsection{Blood Gas Model (SOPAVent)}

The blood gas model SOPAVent (Wang et al., 2006) describes the relationship between the ventilator settings $\left(\mathrm{FiO}_{2}\right.$, PEEP, PIP, RR, Tinsp) and blood gas $\left(\mathrm{PaO}_{2}, \mathrm{PaCO}_{2}\right)$. In the model, the lung is divided into three compartments: The effective compartment (ventilated and perfused), the alveolar dead-space $\left(\mathrm{DS}^{\text {alv }}\right)$ compartment (ventilated but unperfused) and the alveolar shunt $\left(\mathrm{SH}^{\text {alv }}\right)$ compartment (perfused but unventilated). The model is assumed to have an anatomical dead-space and no extra-pulmonary shunt. On inspiration, $\mathrm{CO}_{2}$ gas retained in the anatomical dead-space from previous expiration is assumed to re-enter all ventilated alveoli in proportion to their ventilation.

Following Workman et al. (1965), the volume of mixed expired gas from the alveolar component $\mathrm{V}_{\exp }{ }^{\mathrm{A}}$, is the contribution of the effective compartment $\mathrm{V}_{\exp } \mathrm{E}$, and the alveolar dead-space compartment $\mathrm{V}_{\exp }{ }^{\text {DSalv }}$.

$$
\mathrm{V}_{\exp }{ }^{\mathrm{A}}=\mathrm{V}_{\exp }^{\mathrm{E}}+\mathrm{V}_{\exp }^{{ }^{\text {DS }}{ }^{\text {alv }}}
$$

The ratio of the volume expired from the alveolar dead-space compartment to the volume expired from all ventilated alveoli can obtained as:

$$
\frac{\mathrm{V}_{\exp }{ }^{\mathrm{Da}}{ }^{\text {sav }}}{\mathrm{V}_{\exp }{ }^{\mathrm{A}}}=\frac{\left(\mathrm{P}_{\exp }{ }^{\mathrm{E}}\right)_{\mathrm{CO}_{2}}-\left(\mathrm{P}_{\exp }{ }^{\mathrm{A}}\right)_{\mathrm{CO}_{2}}}{\left(\mathrm{P}_{\exp }{ }^{\mathrm{E}}\right)_{\mathrm{CO}_{2}}-\left(\mathrm{P}_{\exp }{ }^{\mathrm{A}}\right)_{\mathrm{CO}_{2}}+\left(\mathrm{P}_{\exp }\right)_{\mathrm{CO}_{2}}}
$$

Where $\left(\mathrm{P}_{\exp }{ }^{\mathrm{A}}\right)_{\mathrm{CO}_{2}}$ is the partial pressure of $\mathrm{CO}_{2}$ in the alveolar component of expired gas and $\left(\mathrm{P}_{\text {exp }}\right)_{\mathrm{CO}_{2}}$ is the partial pressure of $\mathrm{CO}_{2}$ in the mixed expired gas.

Similarly, the contribution to the arterial blood flow from all perfused alveoli, both ventilated and unventilated is made up of the arterial blood flow from the effective compartment plus the arterial blood flow from the alveolar shunt compartment.

$$
\dot{\mathrm{Q}}_{\mathrm{a}}{ }^{\mathrm{A}}=\dot{\mathrm{Q}}_{\mathrm{a}}{ }^{\mathrm{E}}+\dot{\mathrm{Q}}_{\mathrm{a}}{ }^{\mathrm{SH}} \mathrm{H}^{\mathrm{alv}}
$$

The perfusion of the alveolar shunt compartment as a fraction of the total pulmonary perfusion is obtained from the shunt equation:

$$
\frac{\dot{\mathrm{Q}}_{\mathrm{a}}{ }^{\mathrm{SH}}}{\dot{\mathrm{Q}}_{\mathrm{a}}{ }^{\mathrm{A}}}=\frac{\left(\mathrm{S}_{\mathrm{a}}{ }^{\mathrm{E}}\right)_{\mathrm{O}_{2}}-\left(\mathrm{S}_{\mathrm{a}}\right)_{\mathrm{O}_{2}}}{\left(\mathrm{~S}_{\mathrm{a}}{ }^{\mathrm{E}}\right)_{\mathrm{O}_{2}}-\left(\mathrm{S}_{\mathrm{v}}\right)_{\mathrm{O}_{2}}}
$$

Where $\left(\mathrm{S}_{\mathrm{a}}{ }^{\mathrm{E}}\right)_{\mathrm{O}_{2}}$ is the oxygen saturation contribution to mixed arterial blood from the effective compartment, $\left(\mathrm{S}_{\mathrm{a}}\right)_{\mathrm{O}_{2}}$ is the oxygen saturation from the mixed arterial blood and $\left(\mathrm{S}_{\mathrm{v}}\right)_{\mathrm{O}_{2}}$ is the oxygen saturation of the mixed venous blood.

With the assumption that all ventilated alveoli have equal ventilation and all perfused alveoli have equal perfusion; Workman et al. (1965) defined the fraction of total number of alveoli that are unperfused but ventilated as follows:

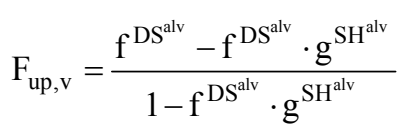

And the fraction of total number of alveoli that are unventilated but perfused as:

$$
\mathrm{F}_{\mathrm{uv}, \mathrm{p}}=\frac{\mathrm{g}^{\mathrm{SH}^{\mathrm{alv}}}-\mathrm{f}^{\mathrm{D} \mathrm{S}^{\mathrm{alv}}} \cdot \mathrm{g}^{\mathrm{SH}}}{1-\mathrm{f}^{\mathrm{DS}} \mathrm{S}^{\mathrm{alv}} \cdot \mathrm{g}^{\mathrm{SH}}}
$$

Where $f^{D S^{a l v}}=\frac{V_{\text {exp }}{ }^{D a l v}}{V_{\text {exp }}{ }^{A}}$ and $g^{D S^{a l v}}=\frac{\dot{Q}_{a}{ }^{S H^{a l v}}}{\dot{Q}_{a}{ }^{A}}$.

Equations (5) and (6) define the link between the gas exchange and lung mechanics models.

\subsection{Lung Mechanics Model}

The lung mechanics model used in this study has been adapted from Hickling (2001). The model is based on the fact that recruitment and derecruitment of the lung alveoli are the principal causes of inflation and deflation respectively. The lung is modelled as multiple units or alveoli which are distributed into compartments characterized by different superimposed pressure (gravitational pressure due to lung weight). In the supine position the superimposed pressure increases from the ventral compartment (independent region) to the dorsal compartment (dependant region). The lung units are described by their compliance curve which gives a nonlinear relationship between the applied pressure and the lung unit volume. The following equation is used to model this relationship (Salazar and Knowles, 1964): 


$$
\mathrm{V}=\mathrm{V}_{0}(1-\exp (-\mathrm{P} \log (2 / \mathrm{h}))
$$

Where $V$ is the lung volume, $V_{0}$ is the maximum volume at high pressure, $P$ is the pressure and $h$ is the half-inflation pressure. In the model, the lung unit can assume only two possible states: recruited (or open) and de-recruited (or closed). These two states are governed only by the Threshold Opening Pressure (TOP) which the critical pressure above which the lung unit pops open and Threshold Closing Pressure (TCP) below which the unit collapses.

The model uses normally distributed TOP and TCP pressures (Crotti et al. (2001)). The Mean ( $\mu$ ) indicates the pressure at which the maximum of recruitment (TOP) and derecruitment (TCP) of the lung units occur, whereas the Standard Deviation (SD) describes the spread of the lung units' population with respect to the TOP and TCP (Yuta et al., 2004). Therefore, $\mu$ and SD may be adjusted to reflect the heterogeneous characteristic of alveoli under different abnormal lung conditions such as ARDS (Table 3) (Markhorst et al., 2004). Fig. 2 illustrates the mechanics of a single lung unit during inflation and deflation.

During inflation (or expiration) when the applied pressure exceeds the TOP, the lung unit pops open and assumes a volume according to (7). During deflation (expiration) when the applied pressure falls below the TCP, the lung unit collapses and its volume drops to zero.

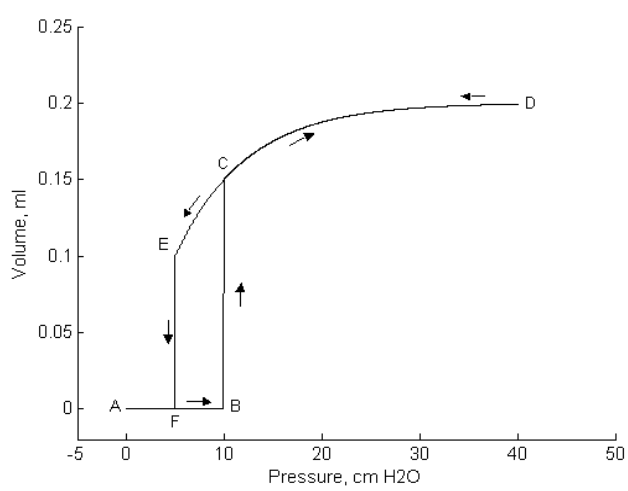

Figure 2: Mechanics of inflation (path $\mathrm{ABCD}$ ) and deflation (path DEFA) of a single lung unit. TOP and TCP coincide with points $\mathrm{B}$ and F respectively.

The lung volume at a given pressure can then be calculated by adding up the contributions of the recruited lung units in the different compartments at that specific pressure. The model parameters used throughout are listed in Table 1 (Hickling, 2001).
Table 1: Lung mechanics model baseline parameters.

\begin{tabular}{|l|c|}
\hline \multicolumn{1}{|c|}{ Parameters } & Value \\
\hline No. of alveoli per compartment $\mathrm{N}_{\text {calv }}$ & 9000 \\
\hline Number of compartments $\mathrm{N}_{\mathrm{c}}$ & 30 \\
\hline Gravitational pressure $\left.\mathrm{P}_{\mathrm{g}} \mathrm{cmH}_{2} \mathrm{O}\right)$ & 0 to 14.5 \\
\hline Lung volume V (litres) & 6 \\
\hline $\mathrm{V}_{0}$ (litres) & 3.8 \\
\hline $\mathrm{h}$ & 5 \\
\hline
\end{tabular}

\subsection{EIT Model}

A typical EIT system uses a set of 16 electrodes attached to the surface of the thorax to inject a small alternating current and record the resulting voltages to reconstruct a cross-sectional image of the internal distribution of the conductivity or resistivity. The quality of the reconstructed images depends on (i) the number of electrodes and data collection strategy and (ii) the reconstruction algorithm employed. The most popular data collection strategy is the so-called adjacent or four-electrode where current is applied to an adjacent pair of electrodes and the resulting voltages between the remaining 13 pairs of electrodes are measured. The type of reconstruction algorithm ranges from a simple linearised singlestep method to a computationally intensive iterative techniques. Mathematically, the problem of recovering the conductivity or resistivity within the body from the boundary measurements of currents and potentials is a nonlinear inverse problem and severely ill-posed. The EIT problem is often approximated by Laplace's equation and Newman type boundary conditions given by (8) as long as the frequency is in the range of $0-10 \mathrm{kHz}$ in which biological tissue exhibits distinct conductivity values (Brown et al., 1985). However, solutions to the full Maxwell's equations have also been investigated (Soni et al. 2006).

$$
\begin{aligned}
& \nabla \cdot(\sigma \nabla \mathrm{u})=0 \\
& \sigma \frac{\partial \mathrm{u}}{\partial \overrightarrow{\mathrm{n}}}=\left\{\begin{array}{rr}
\overrightarrow{\mathrm{J}} & \text { under the electrodes } \\
0 & \text { elsewhere }
\end{array}\right.
\end{aligned}
$$

Where $\sigma$ is the conductivity, $u$ is the potential, $\vec{J}$ is the density of the injected current and $\vec{n}$ is the normal vector to the surface. A systematic approach for solving the reconstruction problem is to solve the forward problem which consists of finding a unique effect (voltages) resulting from a given cause (currents) via a mathematical or physical model (conductivity distribution). The process of 
recovering the conductivity distribution within the body from the applied currents and measured boundary potentials is known as the inverse problem in EIT. There exist two approaches for solving the image reconstruction problem in EIT. Static reconstruction produces an image of the absolute conductivity distribution of the medium based on one set of measurements. Dynamic or difference imaging attempts to recover the change in resistivity based on measurements made at two different time periods.

In this paper, difference imaging was used and the finite element (FE) method was employed for the numerical solution of equation (8) by subdividing a $2 \mathrm{D}$ cross-section of the thorax into a finite number of triangular elements. The electric potential is expressed as a linear combination of the nodal basis functions. Each element is assumed to be homogenous and having the same conductivity. The FE model used to simulate the subject's crosssection of the thorax (adapted from Adler and Lionheart, 2006) was divided into four regions of different conductivities which were fixed to their basal values except those of the left and right lung that were updated based on the instantaneous lung volume generated from the lung mechanics model.

The relationship between changes in the basal conductivity of the lungs and the inspired fraction of air is described by a parametric model. The data used to derive this model were obtained from EIT and spirometry measurements recorded from a human subject during a respiratory cycle (Smulders and van Oosterom, 1992). The left $\left(\sigma_{L}\right)$ and right $\left(\sigma_{R}\right)$ lung relative conductivities estimated from a thorax model for different inspiration levels are listed in Table 2. The inspiration fraction $F$ is defined as: $\mathrm{F}=\left(\mathrm{V}_{\text {insp }}-\mathrm{V}_{\min }\right) /\left(\mathrm{V}_{\max }-\mathrm{V}_{\min }\right)$, where $\mathrm{V}_{\text {insp }}$ represents the tidal volume, $\mathrm{V}_{\min }$ and $\mathrm{V}_{\max }$ are respectively the minimum and maximum volumes assumed during tidal breathing.

Table 2: Left and right lung conductivities $\left(\sigma_{L}, \sigma_{R}\right)$ for different inspiration levels $(F)$.

\begin{tabular}{|c|c|c|}
\hline $\mathrm{F} \mathrm{( \% )}$ & $\sigma_{\mathrm{L}}$ & $\sigma_{\mathrm{R}}$ \\
\hline 0 & 0.8 & 0.8 \\
\hline 20 & 1.0 & 0.8 \\
\hline 40 & 0.9 & 0.7 \\
\hline 60 & 0.7 & 0.6 \\
\hline 80 & 0.5 & 0.6 \\
\hline 100 & 0.4 & 0.5 \\
\hline
\end{tabular}

In this simulation study, the back-projection algorithm (Brown et al., 1985) was used for image reconstruction. The image reconstruction process is illustrated in Fig. 3. At each pressure step, the calculated lung volume is used to set the left and right lung conductivities on the FE thorax model. EIT data (assumed to be the real measurements) are then generated using adjacent drive patterns with an injected current of $5 \mathrm{~mA}$ and matched with the model predicted data set using the backprojection matrix until some precision is reached.

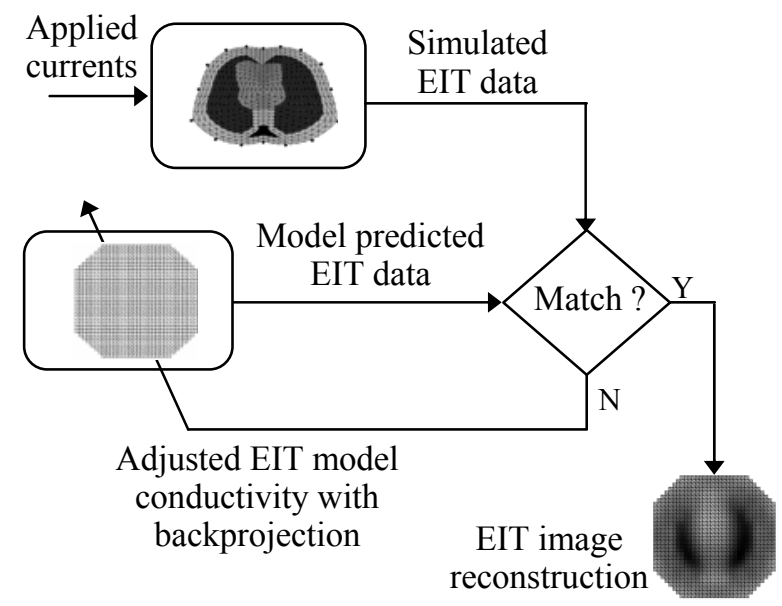

Figure 3: Image reconstruction based on the backprojection.

\section{SIMULATION STUDIES}

In ARDS, the lungs become stiffer and the lung units tend to open and collapse at higher pressures. To reproduce these conditions in the model, $\mu$ and SD which are related to TOP and TCP pressures were given the values shown in Table 3 (Markhorst et al., 2004).

Table 3: Simulated ARDS scenarios.

\begin{tabular}{|l|l|l|l|r|}
\hline $\begin{array}{c}\text { Degree } \\
\text { of } \\
\text { ARDS }\end{array}$ & $\begin{array}{c}\text { TOP } \\
\left(\mathrm{cmH}_{2} \mathrm{O}\right)\end{array}$ & $\begin{array}{c}\mathrm{TCP} \\
\left(\mathrm{cmH}_{2} \mathrm{O}\right)\end{array}$ & $\begin{array}{c}\text { FRC } \\
(\text { litres } \\
)\end{array}$ & $\begin{array}{c}\mathrm{F}_{\mathrm{uv}, \mathrm{p}} \\
(\%)\end{array}$ \\
\hline normal & $4.5 \pm 2$ & $2 \pm 2$ & 2.4 & 0 \\
\hline mild & $10 \pm 2.9$ & $2.5 \pm 2.4$ & 2.2 & 15 \\
\hline moderate & $14.5 \pm 3.8$ & $4.5 \pm 2.9$ & 1.8 & 25 \\
\hline severe & $24.5 \pm 4.8$ & $13 \pm 3.8$ & 1.5 & 35 \\
\hline
\end{tabular}

Estimated values for FRC under the degrees of ARDS conditions considered are listed in Table 3 . It is worth noting that, the amount of collapsed alveoli associated with the shunt fraction are taken into account in the model.

In this initial simulation study, the estimated values for $F_{u v, p}$ (Table 3), expressed as a percentage of the a priori known total number of alveoli, are used to 
simulate the number of collapsed alveoli in these ARDS scenarios.

A tidal breathing cycle is simulated by traversing up (inflation) and then down (deflation) the airway pressure range in small steps from $0 \mathrm{cmH}_{2} \mathrm{O}$ to PIP $=40 \mathrm{cmH}_{2} \mathrm{O}$ and then back from PIP to $0 \mathrm{cmH}_{2} \mathrm{O}$ respectively. Fig. 4 shows the Pressure-Volume (PV) curves obtained for healthy and ARDS affected lungs.
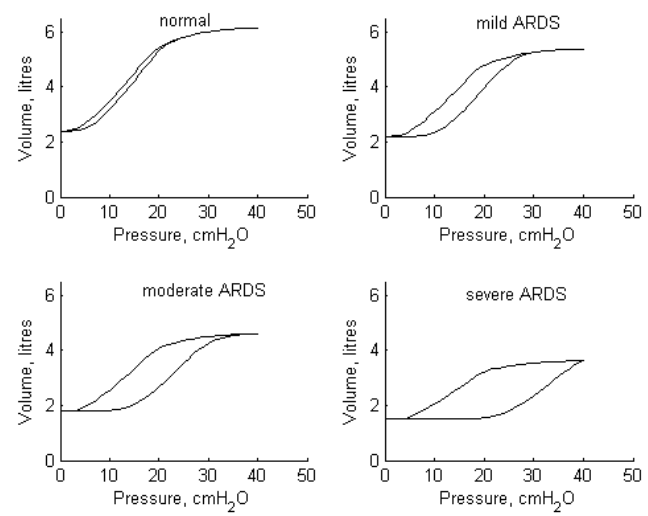

Figure 4: PV curves for the simulated normal (healthy) and ARDS conditions (mild, moderate and severe).

The simulated ARDS scenarios presented in Table 3 are reproduced on the physiological model where the shunt fraction is assumed to quantify the fraction of collapsed alveoli $\mathrm{F}_{\mathrm{uv}, \mathrm{p}}$ (Smith et al., 2005). Figs. 5 and 6 show the sequence of reconstructed images during a breathing cycle (progressing from left to right and top to bottom) for simulated mild and moderate ARDS scenarios respectivelv.

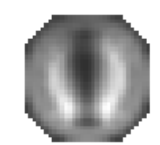

1

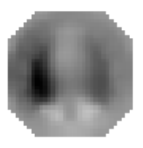

5

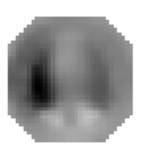

9

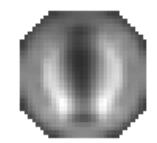

2

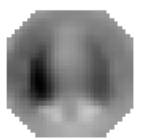

6

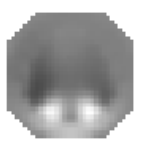

10

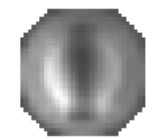

3

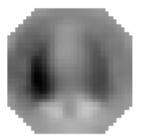

7

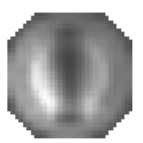

11

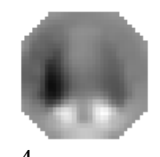

4

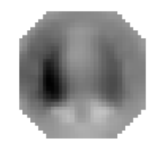

8

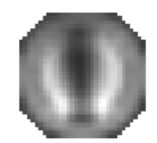

12
Figure 5: Reconstructed images for mild ARDS scenario during a breathing cycle.

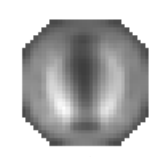

1

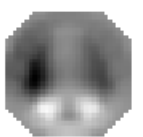

5

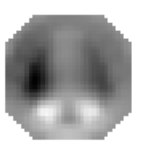

9

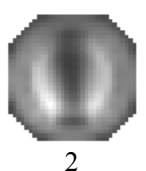

2
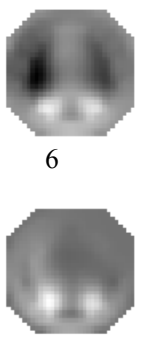

10

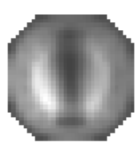

3

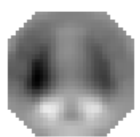

7

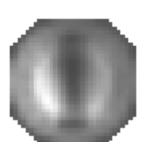

11

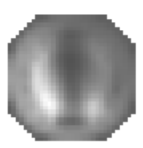

4

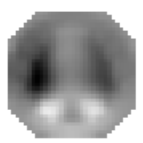

8

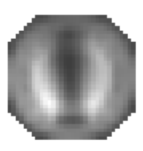

12
Figure 6: Reconstructed images for moderate ARDS scenario during a breathing cycle.

The patient's blood gas model SOPAVent has been validated in a previous study with clinical data gathered from a group of ICU patients (Wang et al., 2007). Table 4 gives the ventilator and model parameters relating to one of the patients.

Table 4: Ventilator settings and SOPAVent predictions.

\begin{tabular}{|l|l|l|l|}
\hline $\begin{array}{l}\mathrm{FiO}_{2} \\
(\%)\end{array}$ & $\begin{array}{l}\mathrm{PEEP} \\
\left(\mathrm{cmH}_{2} \mathrm{O}\right)\end{array}$ & $\begin{array}{l}\mathrm{RR} \\
(\text { breath/min })\end{array}$ & $\begin{array}{l}\mathrm{PIP} \\
\left(\mathrm{cmH}_{2} \mathrm{O}\right)\end{array}$ \\
\hline 65 & 10 & 14 & 28 \\
\hline
\end{tabular}

\begin{tabular}{|l|l|l|l|}
\hline \multicolumn{2}{|c|}{ Estimated parameters } & \multicolumn{2}{c|}{ Predicted blood gases } \\
\hline Shunt & 31.8 & $\mathrm{PaO}_{2}(\mathrm{kPa})$ & 10.3 \\
\hline Dead space & 26 & $\mathrm{PaCO}_{2}(\mathrm{kPa})$ & 5.4 \\
\hline $\mathrm{CO}$ (litres) & 7.4 & & \\
\hline $\mathrm{VCO}_{2}(\mathrm{ml} / \mathrm{min})$ & 138.9 & & \\
\hline $\mathrm{VO}_{2},(\mathrm{ml} / \mathrm{min})$ & 173.6 & & \\
\hline
\end{tabular}

The shunt fraction and relative dead-space in Table 4 have been assumed here to approximate the alveolar shunt $\left(\mathrm{SH}^{\mathrm{alv}}\right)$ and alveolar dead-space $\left(\mathrm{DS}^{\text {alv }}\right)$ respectively and are used to update equations (5) and (6) in the physiological model. The model is cycled through a tidal expiration from FRC to a tidal inspiration and the results are shown in Fig. 7. The fraction of collapsed alveoli obtained from (6) was $25.72 \%$ therefore the lungs were ventilated with over 200,000 alveoli. Fig. $7 \mathrm{~b}$ shows the collapsed lung regions assumed in the model to occur in the dependant sections of the lungs subjected to the gravitational pressure. 


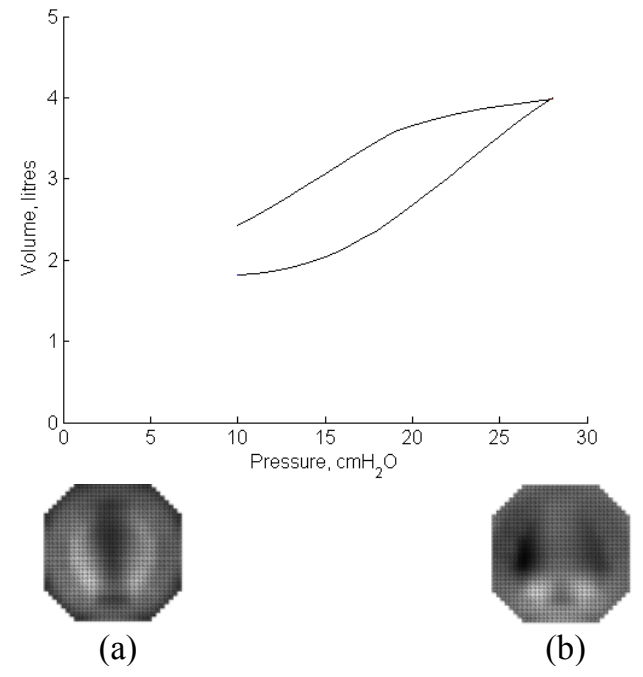

Figure 7: PV curve and EIT images showing end expiration (a) and end inspiration (b) reproduced from this patient's data.

\section{CONCLUSIONS}

EIT is an established monitoring technique with the potential of becoming a valuable bedside tool for the assessment of the pulmonary function in ICUs. EIT is also capable of tracking local changes in pulmonary air contents and thus, can be used to continuously guide the titration of ventilation in ARDS patients whilst minimising the risk of VILI.

A physiological model which combines a blood gas model with a model of lung mechanics has been developed and used to demonstrate the principles of EIT image reconstruction on simulated scenarios of ARDS lungs under mechanical ventilation. The model leads to a good understanding of respiratory physiology in ARDS affected lungs. After its validation against clinical data recorded on realpatients, the model can therefore be used to evaluate a new EIT-based decision support system for effective therapy which is currently being developed by the Sheffield Research Group.

\section{ACKNOWLEDGMENT}

The authors gratefully acknowledge the financial support of the UK Engineering and Physical Sciences Research Council (EPSRC) under Grant EP/520807/1.

\section{REFERENCES}

Adler, A. and Lionheart, W.R.B., 2006. Uses and abuses of EIDORS: an extensible software base for EIT, Physiology Measurement, 27, S25-S42. (Available: http://eidors3d.sourceforge.net/).

Brown, B.H., Barber, D.C. and Seager, A.D., 1985. Applied potential tomography: possible clinical applications, Clin Phys Physiol Mea., 6, 109-121.

Brown, B.H. and Barber D.C., 1992. Electrical impedance tomography, Clinical Physics and Physiological Measurement, Vol. 13, Suppl. A, the Institute of Physical Sciences in Medicine, York.

Crotti, S., Mascheroni, D., Caironi, P., Pelosi, P., Ronsoni, G., Mondino, G.M., Marini, J.J. and Gattinoni, L., 2001. Recruitment and derecruitment during acute respiratory distress syndrome, Am J Respir Crit Care Med, 164, 131-140.

Frerichs, I., 2000. Electrical impedance tomography (EIT) in applications related to lung and ventilation: A review of experimental and clinical activities, Physiol Meas, 21, R1-R21.

Hickling, K.G., 2001. Best compliance during a decremental, but not incremental, positive endexpiratory pressure trial is related to open-lung positive end-expiratory pressure: A mathematical model of acute respiratory distress syndrome lungs. Am J of Respir Crit Care Med, 163, 69-77.

Hinz, J., Moerer, O., Newmann, P., Dudykevych, T., Hellige, G. and M. Quintel, M., 2005. Effect of positive end-expiratory-pressure on regional ventilation in patients with acute lung injury evaluated by electrical impedance tomography, European J of Anaesthesiology, 22, 817-825.

Levy, M.M., 2002. Optimal PEEP in ARDS: Changing concepts and current controversies, Critical Care Clinics, 18(1), 15-33.

Markhorst, D.G., van Genderingen, H.R. and van Vught, A.J., 2004. Static pressure-volume curve characteristics are moderate estimators of optimal airway pressures in a mathematical model of (primary/pulmonary) acute respiratory distress syndrome," Intens Care Med, 30, 2086-2093.

Panoutsos, G., Mahfouf, M., Brown, B.H. and Mills, G.H., 2007. Electrical impedance tomography (EIT) in pulmonary measurement: a review of applications and research," Proceedings of the $5^{\text {th }}$ IASTED International Conference: biomedical engineering, Innsbruck, Austria, 221-230.

Putensen, C., Wrigge, H., and Weiler, N., 2007. Electrical impedance tomography guided ventilation therapy, Current Opinion in Critical Care, 13(3), 344-350.

Salazar, E. and Knowles, J.H., 1964. Analysis of the pressure-volume characteristics of the lungs, $J$ of Appl Physiol, 19, 97-104. 
Smith, B.W., Rees, S., Tvorup, J., Christensen, C.G. and Andreassen, S., 2005. Modelling the influence of the pulmonary pressure-volume curve on gas exchange, Proceedings of the $27^{\text {th }}$ IEEE Annual Conf on Engineering in Medicine and Biology, Shangai, China.

Smulders, L.A. and van Oosterom, A., 1992. Application of electrical impedance tomography to the determination of lung volume, Clin Phys Physiol Meas, 13, 167-170.

Soni, N.K., Paulsen, K.D., Dehghani, H. and Hartov, A., 2006. A 3-D reconstruction algorithm for EIT planner electrode arrays, IEEE Trans. Medical Imaging, 25(1), 55-61.

Tremblay, L.N. and Slutsky, A.S., 2006. Ventilatorinduced lung injury: from the bench to the bedside, Intensive Care Med, 32, 24-33.

Victorino, J.A., Borges, J.B., Okamoto, V.N., Matos, G.F.J, Tucci, M.R., Caramez, M.P.R., Tanaka, H., Sipmann, F.S., Santos, D.C.B., Barbas, C.S.V., Carvalho, C.R.R. and Amato, M.B.P., 2004. Imbalances in regional lung ventilation: A validation study on electrical impedance tomography, Am J Respir Crit Care Med, 169, 791-800.

Wang A., Mahfouf M. and Mills G.H.., 2006. A continuously updated hybrid blood gas model for ventilated patients. The $6^{\text {th }}$ IFAC Symposium on Modelling and Control in Biomedical Systems, Reims, France.

Wang A., Panoutsos, G., Mahfouf M. and Mills G.H., 2007. An improved blood gas intelligent model for mechanically ventilated patients in the intensive care unit. The $5^{\text {th }}$ IASTED International Conference on Biomedical Engineering, Innsbruck, Austria.

Workman, J.M., Penman, W.B., Bromberger-Barnea, B., Perut, S. and Riley, R.L., 1965. Alveolar dead space, alveolar shunt, and transpulmonary pressure. Journal of Applied Physiology, 20, 816-824.

Yuta, Y., Chase, J.G., Shaw, G.M. and Hann, C., 2004. Dynamic models of ARDS lung mechanics for optimal patient ventilation, Proceedings of the $26^{\text {th }}$ Annual International Conference: of the IEEE EMBS San Francisco, USA, 861-864. 